\title{
Congenital Heart Disease and the Liver
}

\author{
Moira B. Hilscher, M.D., and Patrick S. Kamath, M.D.
}

Survival of patients with complex congenital heart disease (CHD) has improved as a result of successful reparative surgeries including the Fontan procedure, which is considered the definitive palliation for patients with single-ventricle physiology. ${ }^{1}$ The Fontan procedure creates an anastomosis between the vena cavae or right atrium (RA) and the pulmonary arteries (Fig. 1), and allows systemic venous blood to bypass a pumping chamber when returning to the lungs. As a result, the Fontan physiology is characterized by elevated central venous pressure and low cardiac output. ${ }^{2}$ Liver complications in patients with CHD are common and may culminate in cirrhosis and hepatocellular carcinoma (HCC). Implementation of evidence-based surveillance and management strategies is therefore essential in this population. ${ }^{3}$

Hepatic dysfunction in patients with CHD is multifactorial and may be caused by the primary cardiac defect or palliative procedures. ${ }^{4}$ Several of the physiological derangements inherent in the Fontan circulation predispose to liver damage, including passive venous congestion, low cardiac output, and hypoxia. In addition, the prevalence of hepatitis $C$ infection is greater among patients with CHD who received blood transfusions or underwent cardiac surgery prior to the implementation of high-sensitivity tests for hepatitis C virus screening in 1992. ${ }^{5,6}$

The clinical manifestations of liver disease in Fontan patients are variable. Patients with hepatic congestion may experience ascites, hepatomegaly, and jaundice. Common laboratory findings include predominantly indirect hyperbilirubinemia, ${ }^{7}$ hypoalbuminemia, and prolongation of the prothrombin time. Aminotransferase elevation occurs in approximately one-third of patients with hepatic congestion and is generally mild, within three to five times the upper limit of normal. The clinical manifestations of cirrhosis are similarly variable. Patients with compensated cirrhosis may be asymptomatic, whereas patients with decompensated cirrhosis may present with ascites, hepatic encephalopathy, jaundice, variceal bleeding, or HCC.

The diagnosis of cirrhosis requires a combination of supportive clinical, laboratory, and radiological features. Although several laboratory-based scoring systems exist to determine the likelihood of cirrhosis, such as the Fibrosis-4 score ${ }^{8}$ or aspartate aminotransferase-to-platelet ratio

Abbreviations: AFP, alpha-fetoprotein; CHD, congenital heart disease; CT, computed tomography; FNH, focal nodular hyperplasia; HCC, hepatocellular carcinoma; IVC, inferior vena cava; MRE, magnetic resonance elastography; MRI, magnetic resonance imaging; PA, pulmonary artery; RA, right atrium; SVC, superior vena cava.

From the Division of Gastroenterology and Hepatology, Mayo Clinic, Rochester, MN.

Potential conflict of interest: Nothing to report.

Received November 6, 2018; accepted March 15, 2019.

View this article online at wileyonlinelibrary.com

C 2019 by the American Association for the Study of Liver Diseases 

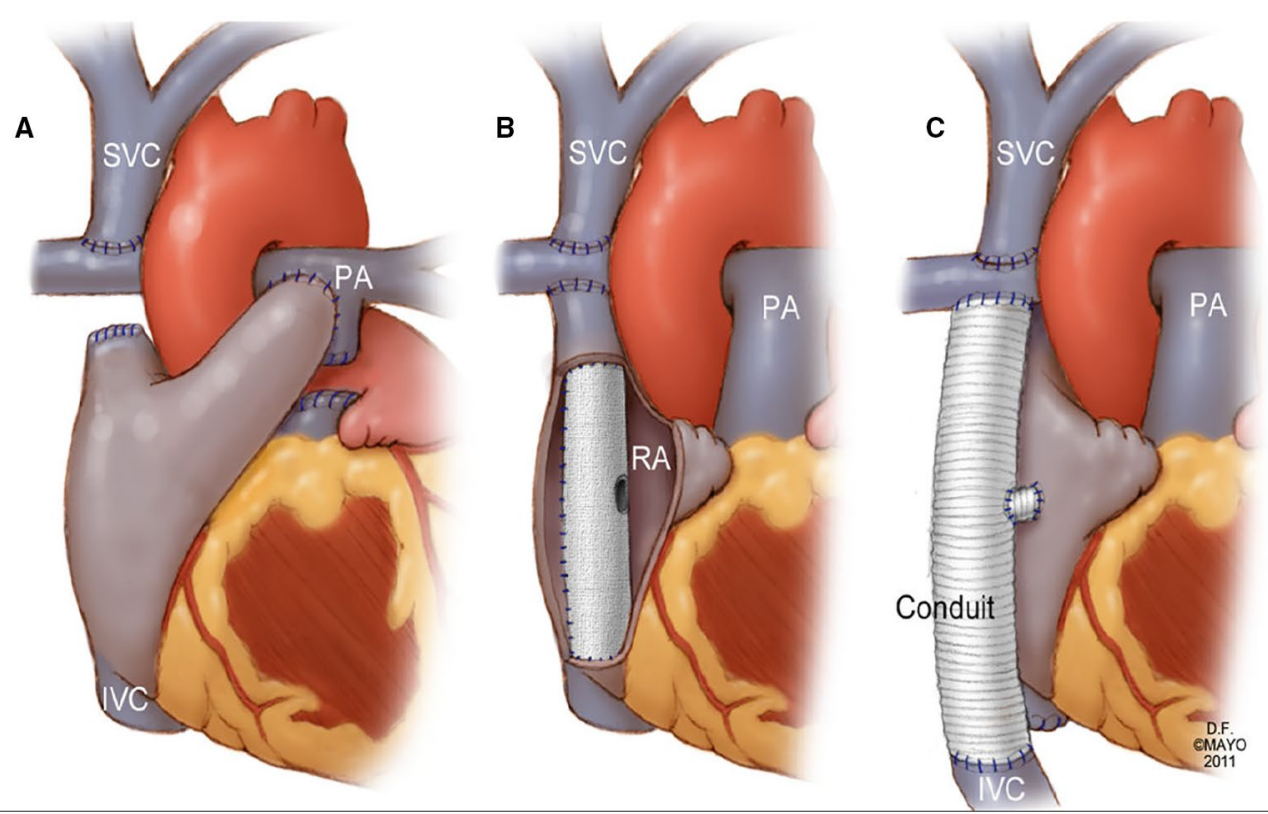

FIG 1 Anatomic variants of Fontan anatomy. (A) A bidirectional cavopulmonary shunt that entails ligation of the superior vena cava (SVC) to the right pulmonary artery (PA) is depicted. The inferior vena cava (IVC) is anastomosed to the PA to supply blood to the lungs. (B) Fontan with an intra-atrial conduit that channels blood from the IVC to the PA through the RA. Blood from the SVC is channeled to the PA. (C) An extracardiac Fontan uses a conduit from the IVC to the right PA with anastomosis of the SVC to the PA. Reprinted from SK Asrani, NS Asrani, DK Freese, SD Phillips, CA Warnes J Heimbach, and PS Kamath. Congenital heart disease and the liver. Hepatology 2012 Sept 56(3); 1160-1169, used with permission of Mayo Foundation for Medical Education and Research. All rights reserved.

index ${ }^{9}$ these models have yet to be validated in the Fontan population. The radiological appearance of the liver on computed tomography (CT) or ultrasound can help to estimate the extent of injury and likelihood of cirrhosis. Liver biopsy remains the gold standard in diagnosing cirrhosis but has several inherent limitations, including cost, sampling error, and risk for adverse events including pain and bleeding. Radiological assessment of liver stiffness with either ultrasound-based shear wave elastography or magnetic resonance elastography (MRE) is frequently used in the staging of fibrosis. ${ }^{10,11}$ The presence of a cardiac pacemaker or implantable defibrillator may complicate MRI in this patient population. In addition, the diagnosis of cirrhosis on the basis of increased stiffness requires caution in patients with $\mathrm{CHD}$ and Fontan physiology because hepatic congestion also elevates liver stiffness. ${ }^{10}$ However, recent studies suggest that liver stiffness scores derived from MRE in patients with Fontan physiology correlate well with liver disease severity as assessed by Model for End-Stage Liver Disease score. In addition, patients with rapid progression of liver stiffness scores on MRE had increased adverse outcomes, including death, listing for heart-liver transplant, and initiation of palliative care. ${ }^{12}$ MRE remains unable to differentiate fibrosis from congestion in this population, however.

Chronic congestion predisposes to the development of both benign nodules, such as focal nodular hyperplasia $(\mathrm{FNH})$, and malignant liver lesions, such as HCC. ${ }^{13,14}$ The congested liver demonstrates parenchymal abnormalities on imaging that can alter the appearance of liver lesions and lead to diagnostic difficulty. As a result, imaging findings alone per the Liver Imaging Reporting and Data System (LIRADS) criteria may not be reliable in diagnosing HCC in patients with chronic hepatic congestion. Use of hepatobiliary contrast agents that are transported by functioning hepatocytes may help differentiate benign lesions, which more often have a homogenous pattern of retention. ${ }^{15-17}$ In contrast, a mass with a mosaic or heterogenous appearance, necrosis, rapid growth over time, or associated venous tumor thrombus is highly suspicious for HCC (Table 1; Fig. 2).

\section{TABLE 1. FEATURES THAT DISTINGUISH BENIGN AND MALIGNANT NODULES IN POST-FONTAN PATIENTS}

Benign

Stable for $\geq 24$ months

$\mathrm{T}_{2}$ hypointensity on MRI
Malignant

Portal venous phase washout

Heterogeneous appearance on imaging Portal thrombus

Tumor necrosis

$\mathrm{T}_{2}$ hyperintensity on MRI

Elevated alpha-fetoprotein

Lesion occurring in cirrhotic liver 


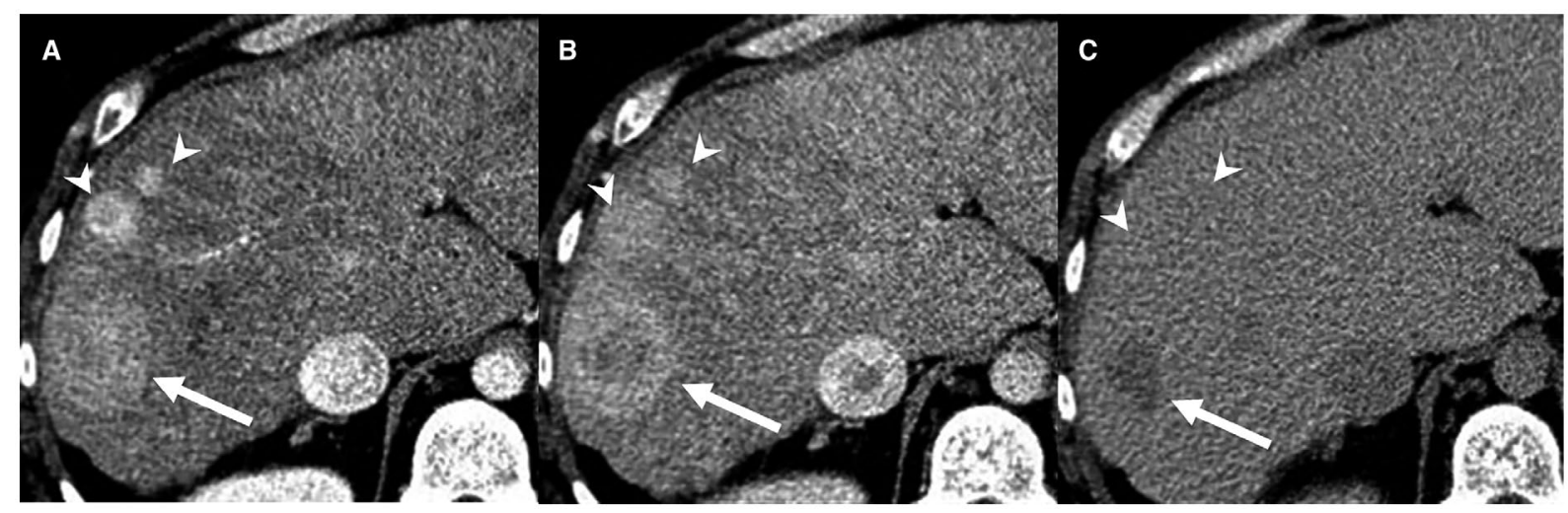

FIG 2 Biopsy-proved hepatocellular carcinoma (A, arrow) in a patient with numerous benign hypervascular nodules, two of which are designated with arrowheads. The HCC is differentiated from the benign nodules by its size and heterogenous washout on portal venous and delayed phase images (B, C, arrows). Reprinted with permission from Abdominal Radiology (New York). ${ }^{13}$ Copyright 2017, Springer Nature.

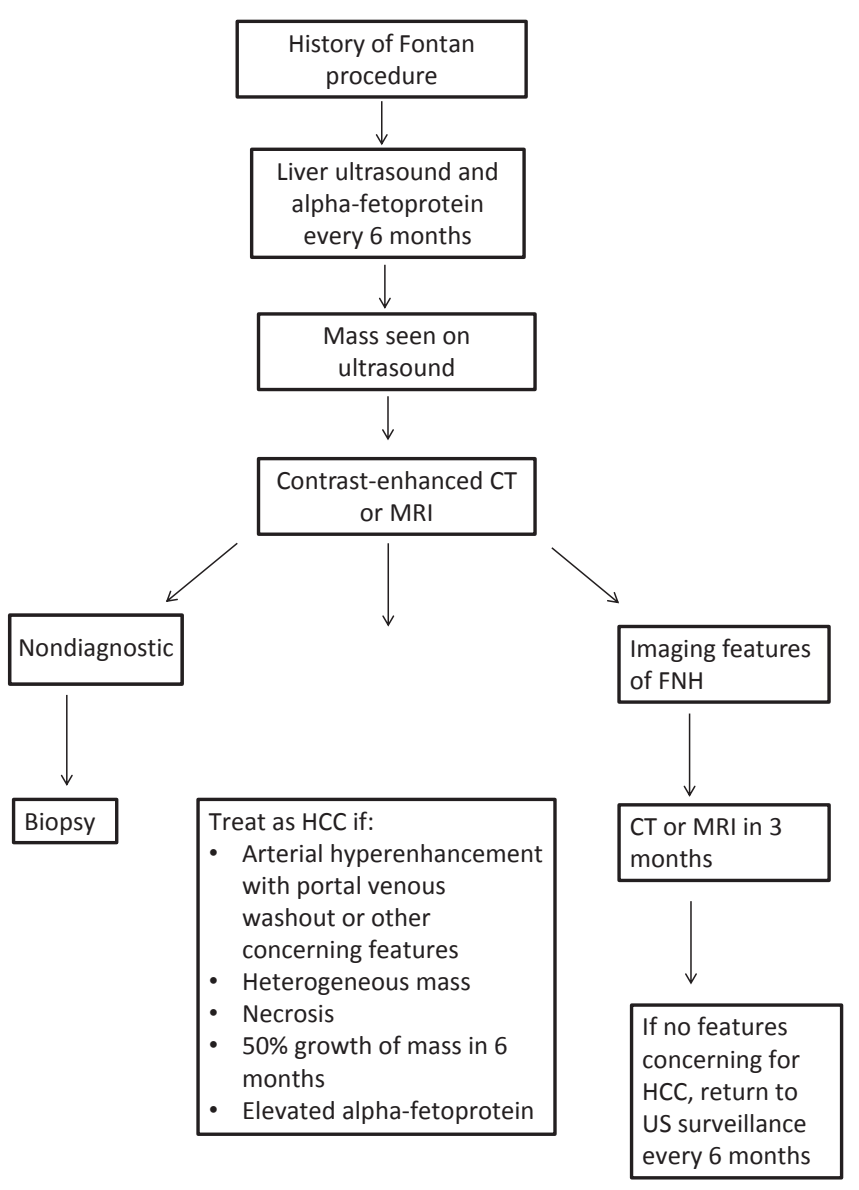

FIG 3 Algorithm for the evaluation and management of liver lesions detected in patients with congestive hepatopathy.

An elevated alpha-fetoprotein (AFP) level further raises suspicion for malignancy. When a hyperenhancing nodule in a congested liver shows delayed phase washout, the presence of a cirrhotic background liver also significantly increases the likelihood of HCC being present. ${ }^{13}$

Post-Fontan patients with cirrhosis should have regular surveillance for HCC with twice-annual ultrasonography and measurement of AFP level (Fig. 3). Contrast-enhanced $\mathrm{CT}$ or magnetic resonance imaging (MRI) should be performed to further evaluate hepatic nodules detected on ultrasound imaging. Hepatic nodules should be diagnosed and treated as HCC if they have characteristic features on either CT or MRI (i.e., arterial hyperenhancement and portal venous washout, heterogeneous appearance, necrosis), or if concomitant AFP levels exceed $200 \mathrm{ng} / \mathrm{mL}$. A mass that shows more than $50 \%$ growth over a 6 -month period is also concerning for HCC. Reimaging with CT or MRI after 3 months is recommended for a mass with characteristics of $\mathrm{FNH}$. If imaging characteristics remain convincing for $\mathrm{FNH}$, then routine surveillance every 6 months can be resumed.

Acknowledgment: The authors thank Dr. Michael Wells for providing $\mathrm{CT}$ images.

\section{CORRESPONDENCE}

Patrick S. Kamath, Division of Gastroenterology and Hepatology, Mayo Clinic, 200 First Street SW, Rochester, MN 55905. E-mail: kamath.patrick@mayo.edu

\section{REFERENCES}

1) Driscoll DJ. Long-term results of the Fontan operation. Pediatr Cardiol 2007;28:438-442.

2) Gewillig M, Goldberg DJ. Failure of the fontan circulation. Heart Fail Clin 2014;10:105-116. 
3) Hilscher $M B$, Johnson JN, Cetta $F$, et al. Surveillance for liver complications after the Fontan procedure. Congenit Heart Dis 2017;12:124-132.

4) Asrani SK, Asrani NS, Freese DK, et al. Congenital heart disease and the liver. Hepatology 2012;56:1160-1169.

5) Wang A, Book WM, McConnell $M$, et al. Prevalence of hepatitis $C$ infection in adult patients who underwent congenital heart surgery prior to screening in 1992. Am J Cardiol 2007;100:1307-1309.

6) Cox DA, Ginde S, Tweddell JS, et al. Outcomes of a hepatitis C screening protocol in at-risk adults with prior cardiac surgery. World J Pediatr Congenit Heart Surg 2014;5:503-506

7) Allen LA, Felker GM, Pocock $S$, et al. Liver function abnormalities and outcome in patients with chronic heart failure: Data from the Candesartan in Heart Failure: Assessment of Reduction in Mortality and Morbidity (CHARM) program. Eur J Heart Fail 2009;11:170-177.

8) Sterling RK, Lissen E, Clumeck N, et al. Development of a simple noninvasive index to predict significant fibrosis in patients with HIV/ HCV coinfection. Hepatology 2006;43:1317-1325.

9) Wai CT, Greenson JK, Fontana RJ, et al. A simple noninvasive index can predict both significant fibrosis and cirrhosis in patients with chronic hepatitis C. Hepatology 2003;38:518-526.

10) Wallihan DB, Podberesky DJ, Marino BS, et al. Relationship of MR elastography determined liver stiffness with cardiac function after Fontan palliation. J Magn Reson Imaging 2014;40: 1328-1335.

11) Serai $S D$, Wallihan $D B$, Venkatesh $S K$, et al. Magnetic resonance elastography of the liver in patients status-post fontan procedure: Feasibility and preliminary results. Congenit Heart Dis 2014;9:7-14.

12) Egbe A, Miranda WR, Connolly HM, et al. Temporal changes in liver stiffness after Fontan operation: Results of serial magnetic resonance elastography. Int J Cardiol 2018;258:299-304.

13) Wells ML, Hough DM, Fidler $\mathrm{JL}$, et al. Benign nodules in post-Fontan livers can show imaging features considered diagnostic for hepatocellular carcinoma. Abdom Radiol (NY) 2017;42:2623-2631.

14) Asrani SK, Warnes CA, Kamath PS. Hepatocellular carcinoma after the Fontan procedure. N Engl J Med 2013;368:1756-1757.

15) Poterucha JT, Johnson JN, Qureshi MY, et al. Magnetic resonance elastography: A novel technique for the detection of hepatic fibrosis and hepatocellular carcinoma after the Fontan operation. Mayo Clin Proc 2015;90:882-894.

16) International Working Party. Terminology of nodular hepatocellular lesions. Hepatology 1995;22:983-993.

17) Motosugi U, Murata S, Shimizu M, et al. Extranodular background liver parenchyma of focal nodular hyperplasia: Histopathological characteristics. Virchows Arch 2009;454:557-562. 